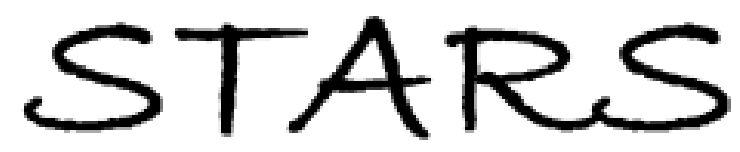

University of Central Florida

STARS

Faculty Bibliography 2000s

Faculty Bibliography

$1-1-2004$

\title{
Neutron transmutation doped far-infrared p-Ge laser
}

E. W. Nelson

University of Central Florida

M. V. Dolguikh

University of Central Florida

A. V. Muravjov

University of Central Florida

E. S. Flitsiyan

University of Central Florida

T. W. Du Bosq

University of Central Florida

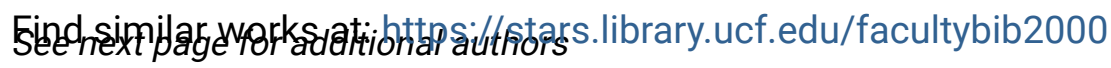

University of Central Florida Libraries http://library.ucf.edu

This Article is brought to you for free and open access by the Faculty Bibliography at STARS. It has been accepted for inclusion in Faculty Bibliography 2000s by an authorized administrator of STARS. For more information, please contact STARS@ucf.edu.

\section{Recommended Citation}

Nelson, E. W.; Dolguikh, M. V.; Muravjov, A. V.; Flitsiyan, E. S.; Du Bosq, T. W.; Peale, R. E.; Kleckley, S. H.; Fredricksen, C. J.; and Vernetson, W. G., "Neutron transmutation doped far-infrared p-Ge laser" (2004).

Faculty Bibliography 2000s. 4608.

https://stars.library.ucf.edu/facultybib2000/4608

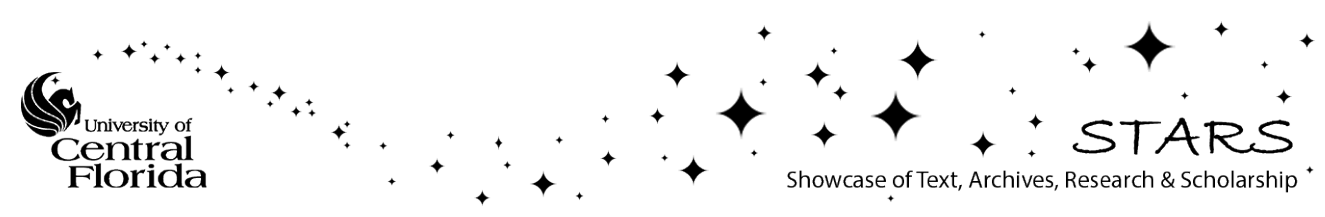




\section{Authors}

E. W. Nelson, M. V. Dolguikh, A. V. Muravjov, E. S. Flitsiyan, T. W. Du Bosq, R. E. Peale, S. H. Kleckley, C. J. Fredricksen, and W. G. Vernetson 


\section{Neutron transmutation doped far-infrared $\mathrm{p}-\mathrm{Ge}$ laser}

Cite as: Journal of Applied Physics 96, 1 (2004); https://doi.org/10.1063/1.1753659

Submitted: 26 January 2004 . Accepted: 02 April 2004 . Published Online: 17 June 2004

E. W. Nelson, M. V. Dolguikh, A. V. Muravjov, E. S. Flitsiyan, T. W. Du Bosq, R. E. Peale, S. H. Kleckley, C. J. Fredricksen, and W. G. Vernetson

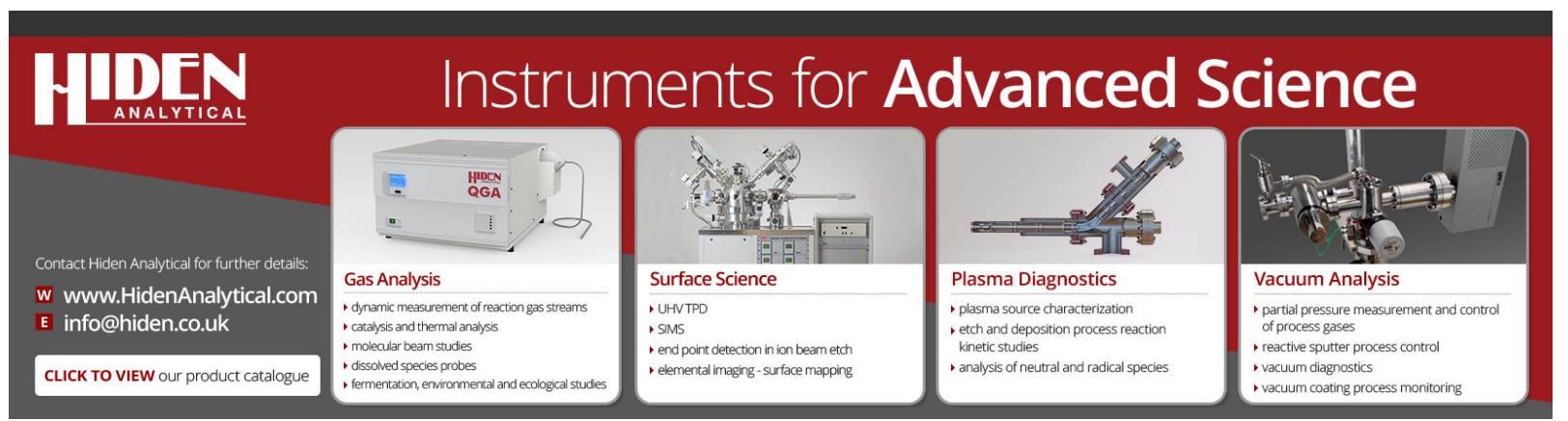




\title{
Neutron transmutation doped far-infrared $\boldsymbol{p}$-Ge laser
}

\author{
E. W. Nelson, M. V. Dolguikh, A. V. Muravjov, ${ }^{\text {a) }}$ E. S. Flitsiyan, T. W. Du Bosq, \\ and R. E. Peale ${ }^{\text {b) }}$ \\ Department of Physics, University of Central Florida, Orlando, Florida 32816 \\ S. H. Kleckley and C. J. Fredricksen \\ Zaubertek, Inc. 1809 E. Broadway St. \#313 Oviedo, Florida 32765 \\ W. G. Vernetson \\ Department of Nuclear and Radiological Engineering, University of Florida, Gainesville, Florida 32611
}

(Received 26 January 2004; accepted 2 April 2004)

\begin{abstract}
A far-infrared $p$-type germanium laser with active crystal prepared from ultra pure single-crystal $\mathrm{Ge}$ by neutron transmutation doping (NTD) is demonstrated. Calculations show that the high uniformity of Ga acceptor distribution achieved by NTD significantly improves average gain. The stronger ionized impurity scattering due to high compensation in NTD Ge is shown to have insignificant negative impact on the gain at the moderate doping concentrations sufficient for laser operation. Experimentally, this first NTD laser is found to have lower current-density lasing threshold than the best of a number of melt-doped laser crystals studied for comparison. (C) 2004 American Institute of Physics. [DOI: 10.1063/1.1753659]
\end{abstract}

\section{INTRODUCTION}

Far-infrared laser emission from hot holes in $p$-Ge, has been intensely investigated. ${ }^{1}$ The mechanism is direct optical transitions between light- and heavy-hole valence subbands at liquid-helium temperatures in crossed electric and magnetic fields. Population inversion is created for certain ratios of electric and magnetic fields. Light holes accumulate in relatively long-lived closed cyclotron orbits, while heavy holes are quickly scattered by optical phonons. Each scattered hole has a finite probability to be scattered to the lighthole subband, which builds a population inversion between subbands. The resulting broad gain spectrum on intersubband transitions allows picosecond far-infrared output pulses $^{2-8}$ or allows broad tunability with high spectral density over the 1.5 to $4.2 \mathrm{THz}$ (50 to $140 \mathrm{~cm}^{-1}$ ) frequency range (70 to 200 $\mu \mathrm{m}$ free-space wavelengths). ${ }^{9-14}$

The traditional pumping scheme for bulk active $p$-Ge crystals is thermally limited to low duty cycles. A burst of optical phonons is generated for each generated far-infrared photon, such that the physical limit of the pumping efficiency is less than $1 \%$, and the active crystal quickly overheats. The resulting increase in lattice absorption and decrease in light hole lifetime terminate the laser emission. With a maximum working temperature around $20 \mathrm{~K}$, the emission pulse lasts less than $10 \mu$ s for $\sim 1 \mathrm{~cm}^{3}$ active volume and $\sim 1 \mathrm{~W}$ output power. The cooling rate in liquid helium of less than $10 \mathrm{~W} / \mathrm{cm}^{2}$ limits the repetition rate to $\sim 100 \mathrm{~Hz}$. In principle, continuous operation could be achieved by reducing the active volume below $1 \mathrm{~mm}^{3},{ }^{15}$ but

\footnotetext{
a) On leave from Institute of the Physics of Microstructures, RAS, Nizhny Novgorod, Russia.

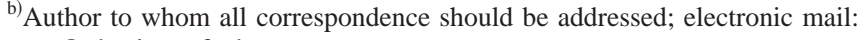
rep@physics.ucf.edu
}

this increases cavity losses, which for a typical gain of only $\sim 0.01-0.1 \mathrm{~cm}^{-1}$ is a critical disadvantage.

To overcome the thermal limitations, it is desirable in $p$-Ge lasers to achieve the highest possible gain. Effort has been devoted to determine the factors responsible for the gain. Active crystal geometry, ${ }^{16}$ crystallographic orientation, ${ }^{17,18}$ doping concentrations, ${ }^{1}$ deep acceptor doping, ${ }^{19}$ uniaxial stress, ${ }^{20-22}$ and optimization of electrodynamic cavities ${ }^{23}$ have been studied. Even moderate improvement of the gain would allow reduced active crystal volume and thus reduced power dissipation and improved cooling efficiency. Still, measured values of gain ranging from ${ }^{24} 0.01$ to ${ }^{25} 0.1 \mathrm{~cm}^{-1}$ fall short of recent predictions, ${ }^{2,26}$ which suggest the possibility of gain as large as $0.4 \mathrm{~cm}^{-1}$ for certain photon wavelengths, applied field strengths, and orientations.

A probable cause of the observed low gain is field inhomogeneity within the active crystal. It is known that a deviation from perpendicularity of applied $\mathbf{E} \times \mathbf{B}$ fields by only $1^{\circ}$ causes a remarkable reduction of gain. ${ }^{1}$ A component of $\mathbf{E}$ parallel to $\mathbf{B}$ (Fig. 1, lower inset) accelerates trapped light holes in the direction of $\mathbf{B}$ until they scatter on optical phonons, decreasing the light-hole lifetime and therefore gain. Hence, field nonuniformity would allow gain as high as the theoretical limit to exist only locally, with losses that must be overcome elsewhere in the medium, giving low values for the observed average gain.

The magnetic field, usually created by a superconducting solenoid or permanent-magnet assembly, ${ }^{27}$ is quite uniform within the active crystal. However, E-field uniformity is degraded under nonlinear conduction conditions by space and surface charge, which appear due to the Hall effect, anisotropic conductivity, and nonuniform doping. Proper choice of orientation for the active crystal eliminates those $\mathbf{E}$ components along $\mathbf{B}$ caused by the Hall effect and anisotropy, but 


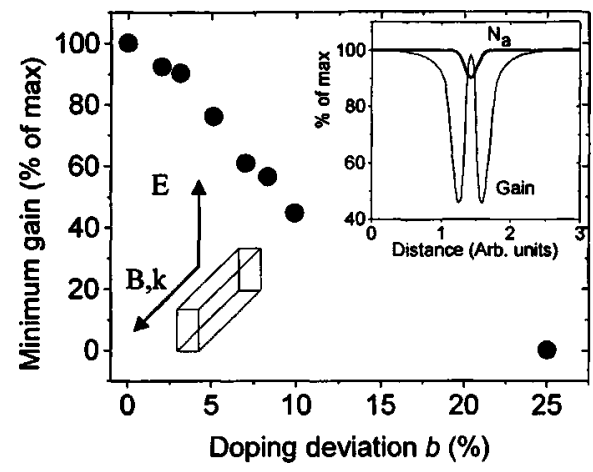

FIG. 1. Lower inset: Schematic of $p$-Ge laser crystal with orientation of applied fields (E, B) and direction of laser output (k). Upper inset: Doping and gain profiles. Symbols: Simulation of peak local gain reduction at 100 $\mathrm{cm}^{-1}$ as a function of doping-anomaly scale.

nonuniform spatial distribution of acceptors is uncontrollable in a given laser rod.

Active $p$-Ge laser crystals are traditionally Ga-doped during melt growth. Room temperature resistivity variations typically exceed $4 \%-10 \%$ over both macroscopic (centimeter) and microscopic (sub-millimeter) length scales. ${ }^{28}$ In the lightly doped ( 35 to $40 \Omega \mathrm{cm}$ ) material required for $p$-Ge lasers, intrinsic carriers significantly contribute to the room temperature resistivity. Hence, variations in acceptor concentration can greatly exceed room-temperature resistivity variations.

This article presents results of neutron transmutation doping (NTD) ${ }^{28-30}$ to produce uniformly doped $p$-Ge laser material. Some preliminary results have been reported in Refs. 31 and 32. Room temperature resistivity variations of NTD Ge over submillimeters to centimeters lengths are less than $0.7 \%{ }^{28}$ The NTD method immerses undoped Ge in the homogeneous neutron flux of a nuclear reactor. Three of the five stable natural $\mathrm{Ge}$ isotopes, ${ }^{70} \mathrm{Ge},{ }^{74} \mathrm{Ge}$, and ${ }^{76} \mathrm{Ge}$ are transmuted by thermal neutrons into ${ }^{71} \mathrm{Ga}$ (shallow acceptor), ${ }^{75} \mathrm{As}$ (shallow donor), and ${ }^{77} \mathrm{Se}$ (double donor), respectively. The remaining two isotopes $\mathrm{Ge}^{72}$ and $\mathrm{Ge}^{73}$, transmute into other stable $\mathrm{Ge}$ isotopes $\mathrm{Ge}^{73}$ and $\mathrm{Ge}^{74}$, respectively. The low doping levels needed for $p$-Ge lasers require less than 1 $\mathrm{h}$ irradiation in a typical reactor. The donors As and Se are produced in lower concentrations than the acceptor $\mathrm{Ga}$, so that NTD Ge is $p$-type with reported compensations $K$ $=N_{d} / N_{a}$ in the range $0.32-0.40,{ }^{29,30}$ where $N_{d(a)}$ is the concentration of donors (acceptors). In the few melt-doped $p$-Ge lasers tested for compensation, the value has been between ${ }^{33}$ 0.07 and $^{34} 0.3$, but the maximum allowable compensation for a $p$-Ge laser is uncertain.

A practical consideration in favor of the NTD process for producing $p$-Ge lasers is that the ultra-pure starting material is readily available from commercial suppliers. Large single crystals of this highly refined germanium are used in gamma-ray detectors. In contrast, suitable melt-doped $p$-Ge is not a standard item of commerce.

Long lived radioactivity can be generated in natural germanium only by activation of impurities, since final transmutation products of natural Ge isotopes are stable and result from rapid decays. Most of the expected impurities in ultra- pure Ge starting material generate isotopes with half-lives from seconds to minutes. Preirradiation polishing with diamond ensures that residual surface impurities are mainly carbon. Diamond powder may contain $\mathrm{Zn}$ as a (removable) impurity, which can lead to radioactive Zn-65. Cleaning of cut laser rods in HF vapor removes surface contamination without introducing metal ions.

\section{THEORETICAL CONSIDERATIONS}

It is important to emphasize that, due to saturation of the current in the strong applied E-fields required for lasing, even moderate doping inhomogeneity dramatically disrupts the $\mathbf{E}$-field homogeneity within the active sample. This drastically reduces the gain because of its strong sensitivity to deviation in angle between local-E and applied-B fields from $90^{\circ}$. To obtain quantitative estimates of this effect, the internal $\mathbf{E}$-field distribution was found by numerically solving the equation

$$
\operatorname{div}(\mathbf{J})=0
$$

in the presence of a doping anomaly using the finite element method, where $\mathbf{J}$ is the current density. A simple ad hoc model of the nonohmic current in the near-saturation regime that qualitatively gives the correct dependence is

$$
\mathbf{J}=\sigma \mathbf{E}^{(1-s)},
$$

with saturation factor $s=0.75$. Effects of the $\mathbf{B}$-field on current redistribution were neglected. A doping anomaly of the form

$$
N_{a} / N_{a 0}=1-b \operatorname{Exp}\left[-r^{2} / R^{2}\right]
$$

was assumed, where $N_{a}$ is the acceptor concentration, $b$ is the anomaly magnitude, and $R$ its direction-dependent length scale. The calculated $\mathbf{E}$-field redistribution was combined with gain versus E-field-deviation results from Monte-Carlo simulation ${ }^{26}$ to determine the spatial gain distribution near the anomaly.

The upper insert in Fig. 1 shows acceptor concentration and gain profiles for a spherical doping anomaly. The gain dependence is plotted for a profile oriented at $45^{\circ}$ to the applied $\mathbf{E}$-field, which corresponds to the strongest gain distortion. The calculations showed that distorting the anomaly from spherical shape distorts the gain profile, but the maximum gain reduction stays approximately the same and depends mainly on the degree of the anomaly $b$. The dependence of the minimal gain versus $b$ is plotted as symbols in Fig. 1. These results are valid for any anomaly scale starting from light hole mean free path $(10 \mu \mathrm{m})$ to the full size of the active crystal.

Although the average gain reduction will depend on the individual pattern of the acceptor distribution, the Fig. 1 dependence characterizes the effect of non-uniform doping. The region with degraded gain always has much larger spatial extent than the doping anomaly. For melt-doped $p-\mathrm{Ge}$ with just $15 \%$ doping inhomogeneity, the gain in certain regions drops threefold. These considerations can explain the observed performance variations for different crystals cut from the same melt-doped boule, which will be demonstrated below. 


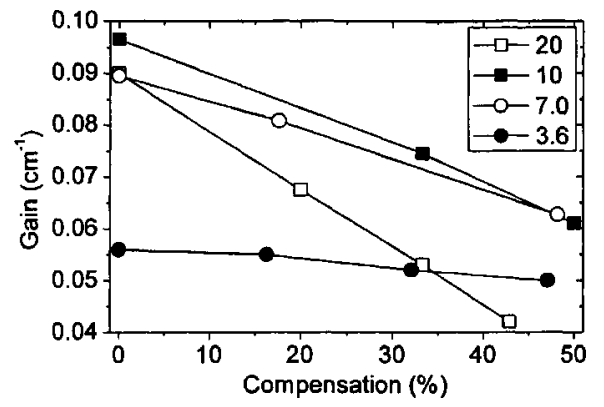

FIG. 2. Monte Carlo calculation of gain at $100 \mathrm{~cm}^{-1}$ vs compensation for different hole concentrations. The legend gives hole concentrations in units of $10^{13} \mathrm{~cm}^{-3}$. Simulation parameters were $E=1.25 \mathrm{kV} / \mathrm{cm}, B=1 \mathrm{~T}$, and $T=10 \mathrm{~K}$.

Monte Carlo simulation ${ }^{26}$ provides predictions for the effect of compensation on gain. Simulation results are presented in Fig. 2, where gain at $100 \mathrm{~cm}^{-1}$ is plotted versus compensation for four different hole concentrations. (These Monte Carlo calculations differ from Ref. 2 by assuming isotropic dispersion and implementing a more accurate approach to impurity and electron-electron scattering. ${ }^{26}$ Thus the gain values in Fig. 2 are smaller than those found in Ref. 2 , where a maximum gain of $0.4 \mathrm{~cm}^{-1}$ was calculated.) At zero compensation, the hole concentration that maximizes the gain is $10^{14} \mathrm{~cm}^{-3}$, which is close to the experimental value of $\sim 7 \times 10^{13} \mathrm{~cm}^{-3}$. ${ }^{1}$ The effect of compensation is in all cases to lower the gain, but this effect becomes less and less pronounced with decreasing hole concentration. At the concentration $3.6 \times 10^{13} \mathrm{~cm}^{-3}$, the effect of compensation is almost negligible.

Hole concentration $p$ may be determined from room temperature resistivity $\rho$ of germanium samples according to

$$
1 / \rho=e\left(\mu_{p} p+\mu_{n} n_{i}^{2} / p\right)
$$

where $e$ is the elementary charge, and $\mu_{n, p}$ are room temperature mobilities of electrons and holes, taken as 3900 and $1900 \mathrm{~cm}^{2} / \mathrm{V}^{*}$ s, respectively. ${ }^{35}$ The value for intrinsic carrier concentration $n_{i}=1.87 \times 10^{13} \mathrm{~cm}^{-3}$ at $23{ }^{\circ} \mathrm{C}$ sample temperature according to Ref. 36 . A complementary measure of $p$ may be deduced from the low temperature saturated current density according to

$$
J_{s}=e v_{s} p
$$

where $v_{s}$ is the average saturated hot hole velocity in Ge at liquid helium temperature. A value of $v_{s}=0.98 \times 10^{5} \mathrm{~m} / \mathrm{s}$ was determined by Monte Carlo simulation with applied fields $E=1 \mathrm{kV} / \mathrm{cm}$ and $B=0$. Compensation $K$ is related to $p$ and $N_{a}$ by

$$
p=\left(N_{a}-N_{d}\right)=N_{a}(1-K) .
$$

An indication of doping homogeneity is saturation current behavior at high electric field, where the dominant scattering mechanism is optical phonon scattering. Simulated saturation curves (Monte Carlo method ${ }^{26}$ ) are presented in Fig. 3 for homogeneous and inhomogeneous crystals. The latter is a rather extreme case, where the crystal length $L$ is divided into two sections, one of length $0.9 \mathrm{~L}$ and concentration $p$, and one with length $0.1 L$ and concentration $0.7 p$. The

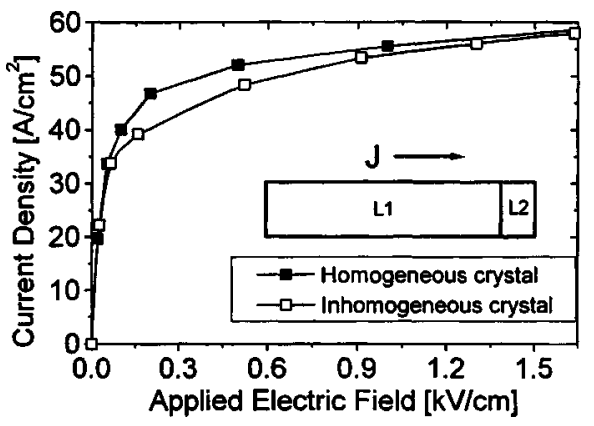

FIG. 3. Monte Carlo calculation of current density as a function of applied electric field for a homogeneous and an inhomogeneous crystal. Inhomogeneity distribution and direction of current are shown schematically in the inset. The total impurity concentration was taken to be $N_{i}=6.7 * 10^{13} \mathrm{~cm}^{-3}$ with $30 \%$ compensation $\left(p=N_{a}-N_{d} \sim 3.6 \times 10^{13} \mathrm{~cm}^{-3}\right)$.

inhomogeneous curve was obtained using the average carrier velocity versus electric field, obtained from the Monte Carlo simulation in the homogeneous case, together with the continuity condition for current. The transition between ohmic and saturated regions is sharper for the homogeneous crystal.

\section{EXPERIMENTAL DETAILS}

To determine the statistical dispersion of laser performance resulting from random doping anomalies, 18 laser rods were prepared from $\mathrm{Ge}$ melt-doped with $\mathrm{Ga}$, designated material C. The original boule was $9.9 \mathrm{~cm}$ in length with 3.4 $\mathrm{cm}$ diameter. The vendor (Tydex, St. Petersburg, Russia) specified the axial orientation as [110], the range of resistivity as 34 to $46 \Omega \mathrm{cm}$, and the dislocation density as 1000 $\mathrm{cm}^{-2}$. The radial crystallographic orientations were determined by HeNe laser light scattering from suitably etched surfaces. ${ }^{37}$ The boule was cross cut into two cylinders of length 46.4 and $52.4 \mathrm{~mm}$ using a wire saw. These cylinders were then cut in half longitudinally. A low-speed diamond saw then cut the longer hemi-cylinder into nine laser rods (C1-C9), as shown in Fig. 4. Nine more laser rods (C10C18) were cut from the shorter hemicylinder. Table I lists dimensions of the laser rods studied.

Laser cavities were formed using $\mathrm{SrTiO}_{3}$ mirrors attached to the active sample end faces. The back mirror covered one face of the active crystal. The output mirror on the opposite face was of a smaller area to allow radiation to escape around the mirror edge. E-field pulses of 1-2 $\mu \mathrm{s}$ duration were generated in the laser crystal by applying high voltage with a thyratron pulser to ohmic contacts on lateral surfaces of the crystal. Current through the active crystal was monitored using a fast current probe (Pearson 411). The magnetic field was supplied by a superconducting solenoid in Faraday configuration. Laser emission was detected using a Ge:Ga photoconductor immersed in liquid helium at $4 \mathrm{~K}$ in the same dewar.

For neutron transmutation doping, a rod measuring $\sim 37$ $\times 6 \times 5 \mathrm{~mm}^{3}$ was cut from high purity germanium (Perkin Elmer). The electrically active impurity concentration was specified as $1.02 \times 10^{11} \mathrm{~cm}^{-3}$. Neutral impurities $(\mathrm{Si}, \mathrm{H}, \mathrm{O}, \ldots$, ) may be present in higher concentrations. The rod was rinsed in spectroscopy-grade acetone, etched in HF vapor, and fi- 


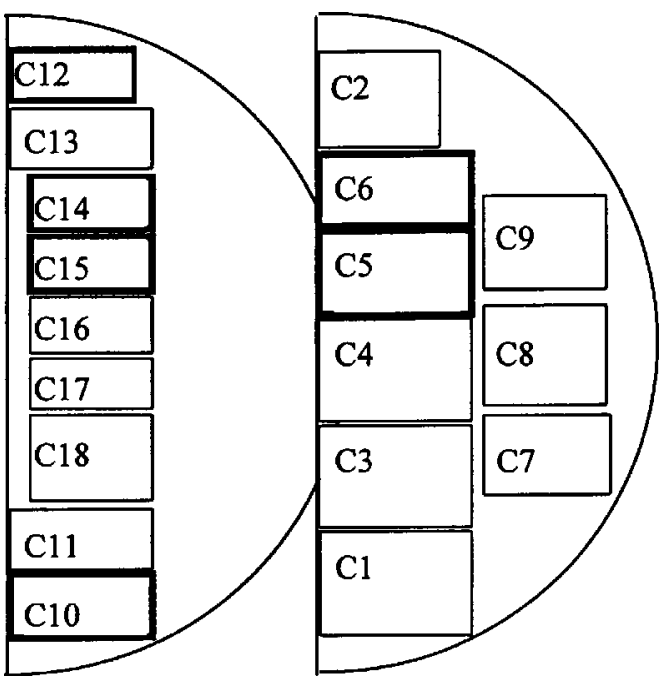

FIG. 4. Crystals cut from a commercial $p$-Ge boule of melt-doped material C. The crystal cross sections are shown to scale and labeled with their Table I designations. The six best performers (lowest $\mathbf{E}$-field thresholds) are highlighted.

nally rinsed in distilled water to remove trace surface contaminants. The rod was wrapped in polyethylene and irradiated in the central vertical port of the University of Florida Training Reactor (Gainesville, Florida) (UFTR, 1.5 $\times 10^{12} \mathrm{n} / \mathrm{cm}^{2} \mathrm{~s}$ thermal neutron flux) for $25 \min$ (becoming material D). Additional fast neutron flux gave an estimated effective thermal neutron flux for the intended reactions of $3 \times 10^{12} \mathrm{n} / \mathrm{cm}^{2} \mathrm{~s}$. After irradiation, radionuclides present in the rods were identified using a high purity germanium detection system. Total emission rate was determined with a Geiger counter (Ludlum-3). Seven days after irradiation at UFTR, the radionuclides detected by $\gamma$-emission spectroscopy in material D were primarily Ge-77, Ge-75, and As- 77 .

TABLE I. Laser rods. Material C is melt doped. Material D is neutron transmutation doped. An asterisk (*) designates nonworking lasers. The dimensions given are the long axis (B-field direction), E-field direction, and direction perpendicular to $\mathbf{E}$, respectively. Crystallographic orientations are $[110] \times[-110] \times[001]$ for these directions for all crystals.

\begin{tabular}{lcc}
\hline \hline Designation & Dimensions $\left[\mathrm{mm}^{3}\right]$ & E-field lasing threshold $(\mathrm{kV} / \mathrm{cm})$ \\
\hline $\mathrm{C} 1$ & $51.8 \times 7.6 \times 5.1$ & 0.78 \\
$\mathrm{C} 2$ & $51.2 \times 7.5 \times 5.2$ & 0.91 \\
$\mathrm{C} 3$ & $51.8 \times 7.6 \times 5.1$ & 1.1 \\
C4 & $51.3 \times 7.5 \times 5.1$ & 1.0 \\
C5 & $51.2 \times 7.6 \times 4.2$ & 0.72 \\
C6 & $51.2 \times 7.6 \times 3.65$ & 0.64 \\
C7 & $51.2 \times 6.35 \times 4.0$ & 0.78 \\
C8 & $51.8 \times 6.1 \times 5.0$ & 0.90 \\
C9 & $51.8 \times 6.05 \times 4.75$ & 1.3 \\
C10 & $45.45 \times 7.0 \times 3.2$ & 0.86 \\
C11 & $45.45 \times 6.95 \times 3.1$ & 0.86 \\
C12 & $45.45 \times 6.0 \times 2.5$ & 0.72 \\
$*$ C13 & $45.45 \times 6.9 \times 3.1$ & - \\
C14 & $45.7 \times 5.9 \times 2.6$ & 0.64 \\
C15 & $45.7 \times 6.0 \times 2.5$ & 0.67 \\
C16 & $45.7 \times 6.1 \times 2.85$ & 0.82 \\
C17 & $45.7 \times 5.9 \times 2.51$ & 0.93 \\
$*$ C18 & $45.7 \times 5.9 \times 4.3$ & - \\
D1 & $17.40 \times 6.16 \times 2.44$ & 0.71 \\
\hline \hline
\end{tabular}

Thirteen days after irradiation, only As-77 was detectable. At that time, the germanium was found to emit $\sim 0.01 \mathrm{mR} / \mathrm{hr}$, which is indistinguishable from background.

The NTD rods were tested for laser action before and after annealing. For initial laser testing, the NTD sample measured approximately $37 \times 6 \times 5 \mathrm{~mm}^{3}$. This crystal was then cut into four parts measuring $18.38 \times 6.16 \times 2.44 \mathrm{~mm}^{3}$ and tested again for lasing prior to annealing. One of these crystals (D1) was annealed $30 \mathrm{~min}$ at $390^{\circ} \mathrm{C}$, repolished, and tested for laser emission. Table I gives its final dimensions.

To obtain information on gallium concentration and compensation, neutron activation analysis (NAA) was performed on samples of materials $\mathrm{C}$ and D. Typical sample mass was between $0.1-0.3 \mathrm{~g}$. Samples were placed into $0.3-\mathrm{ml}$ polypropylene vials and irradiated for $30 \mathrm{~min}$ at Uzbekistan Academy of Science (Tashkent, Uzbekistan) reactor $\left(10 \mathrm{~kW}\right.$, open pool, $3 \times 10^{13}$ thermal neutron $\left./ \mathrm{cm}^{2 *} \mathrm{~s}\right)$. Neutron capture by the ${ }^{71} \mathrm{Ga}$ (which exists in both NTD and melt-doped $p$-Ge $)$ produces the isotope ${ }^{72} \mathrm{Ga}\left(T_{1 / 2}\right.$ $=14.1 \mathrm{~h}$ ), whose $834 \mathrm{keV} \gamma$-line is the Ga signature used for the NAA. After a six-day cooling-off period, which allows the majority of the interfering isotope ${ }^{69} \mathrm{Ge}$ to decay, a Ge:Li detector counted the $\gamma$-ray emission. Relative ${ }^{71} \mathrm{Ga}$ concentration was determined from the ${ }^{72} \mathrm{Ga} 834 \mathrm{keV}$ line strength. In NTD samples, the isotopic abundance of ${ }^{71} \mathrm{Ga}$ is $100 \%$, but the natural abundance of ${ }^{71} \mathrm{Ga}$ in the melt-doped samples is only $39.89 \%$. Therefore, the $834 \mathrm{keV}$ line strength for melt-doped samples was multiplied by the factor 1/0.3989.

For resistivity measurements, a constant current $(<15$ $\mathrm{mA}$ to avoid heating) was applied, and resistance was determined from the measured potential drop. Resistivity was calculated from these data using rod dimensions. Hole concentration $p$ was then found from Eq. (4). Values for $p$ were also determined from the saturated current density according to Eq. (5). Comparison of $p$ versus gallium concentration from neutron activation analysis then provides information about compensation $K$, according to Eq. (6).

To compare the shape of current saturation curves, two identically shaped rods of materials $\mathrm{C}$ and $\mathrm{D}$ were immersed in liquid helium and subjected to microsecond current pulses along their long axis [110]. The experiments were performed in the presence of a small magnetic field $(0.19 \mathrm{~T})$ applied axially.

\section{RESULTS}

Laser generation zones in the space of applied $\mathrm{E}$ and $\mathrm{B}$ fields for melt-doped samples $\mathrm{C} 1-\mathrm{C} 12$ and $\mathrm{C} 14-\mathrm{C} 17$ are presented in Fig. 5. Samples C13 and C18 failed repeatedly to produce laser emission, while all other $\mathrm{C}$ crystals worked during their first trials. A large variation in electric field threshold is observed. Crystal C6 has the lowest electric field and current density thresholds. The latter has the value 74.4 $\mathrm{A} / \mathrm{cm}^{2}$ for $\mathrm{C} 6$.

Far-infrared laser emission from NTD laser crystal D1 was observed only after annealing. The region of observed laser emission in the space of applied electric and magnetic fields is plotted in Fig. 5. The electric field threshold is comparable to the lowest thresholds found in melt-doped mate- 


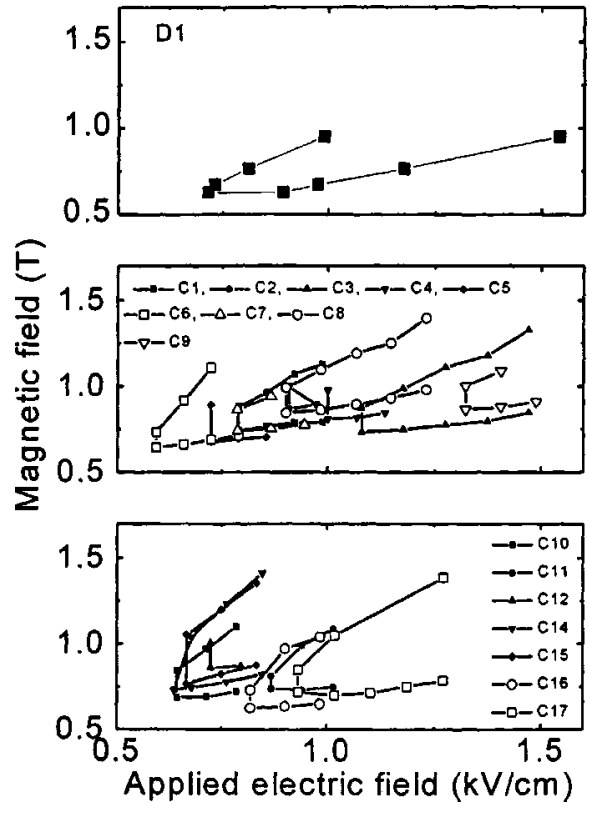

FIG. 5. (top) Zone for observed far infrared laser emission for annealed NTD laser rod D1 in applied field space. (middle) Laser generation zones of $\mathrm{C} 1-\mathrm{C} 9$ and (bottom) $\mathrm{C} 10-\mathrm{C} 17$.

rial $\mathrm{C}$, but the current density threshold is considerably lower at $50.7 \mathrm{~A} / \mathrm{cm}^{2}$.

Saturated current density versus electric field applied via lateral laser contacts was measured for D1 and C6 in liquid helium. For a given crystal, saturation curves for different applied magnetic fields asymptotically approach the same saturated current density $J_{s}$. Using Eq. (5), hole concentrations $p$ are determined from $J_{\mathrm{s}}$ values and plotted against normalized gallium concentration from NAA as open symbols in Fig. 6. Values of $p$ determined from resistivity data and Eq. (4) are plotted as solid symbols in Fig. 6. The crystal D1 has a lower hole concentration than C6 by a factor 1.04 (resistivity data) to 1.5 (current saturation data). Since the $\mathrm{Ga}$ concentration of D1 is higher, its compensation is higher. By calibrating the concentration scale using $p$, the expected compensation of $32 \%-40 \%$ for D1, and Eq. (6), lines of zero

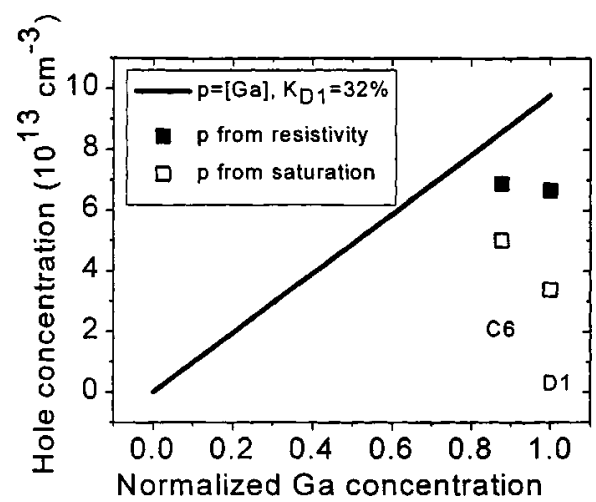

FIG. 6. Hole concentration $p$ as a function of normalized Ga concentration for melt-doped crystal C6 and for annealed NTD crystal D1 as determined by room temperature resistivity (solid symbols) and as determined by saturation current density at $4 \mathrm{~K}$ (open symbols). The zero compensation line is found by calibrating the normalized concentration using the D1 $p$ value from resistivity and assuming $32 \%$ compensation.

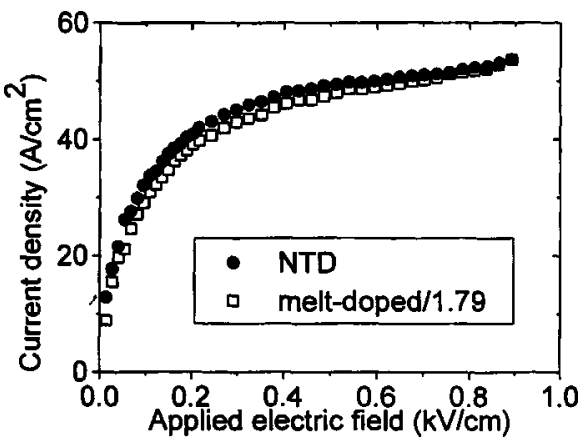

FIG. 7. Current density vs applied electric field for annealed NTD (D) and melt-doped (C) $p$-Ge crystals of identical dimensions. A small longitudinal magnetic field of $0.19 \mathrm{~T}$ is applied to suppress electron injection. Sample temperature was $4 \mathrm{~K}$. The data are scaled to have the same maximum value. The scaling factor is given in the legend.

compensation (or $p=[\mathrm{Ga}]$ ) can be drawn. The straight line in Fig. 6 is the $K=0$ line found using the $p$ values from resistivity data and a $K$ value of $32 \%$ for D1. This suggests that the compensation of the C6 crystal is $\sim 20 \%$. If $\mathrm{K}$ was set to $40 \%$ for D1, the compensation of C6 would be $\sim 30 \%$. Hence, the NTD laser crystal has compensation 1.3 to 1.6 times higher than the best of the melt-doped crystals studied.

Current saturation curves were compared for rods of material $\mathrm{C}$ and annealed $\left(1 \mathrm{~h}\right.$ at $\left.390^{\circ} \mathrm{C}\right)$ material $\mathrm{D}$ having identical geometry and axial current flow (Fig. 7). The transition to saturation is slightly sharper for the NTD crystal than for the melt-doped crystal. The difference is less than the simulated Fig. 4 curves, though it was expected that real crystals would have less extreme inhomogeneity than the simulation model used for Fig. 4. Nevertheless, these data support the assertion that the doping uniformity achieved in the NTD crystal is higher than in the melt-doped crystal studied.

\section{DISCUSSION}

Electric field threshold of the laser operation is a qualitative indicator of laser gain. Thus, Fig. 5 indicates a large variation in the quality of laser rods prepared from the same melt-doped boule and tested under identical conditions. This variability can be attributed to the poor uniformity of lowconcentration doping for melt-doped crystals. Since most of the best performing crystals tend to come from the same portion of each hemicylinder (Fig. 3), there is likely substantial doping inhomogeneity over centimeter length scales. Hence, a typical melt-doped $p$-Ge boule will have low yield of good laser rods.

Current density threshold for D1 is a factor 1.5 times smaller than that found for the best of the C crystals (C6). This positive result occurs despite a much smaller active volume for D1. The larger number of round trips required to build up laser oscillation therefore implies that the low D1 threshold occurs despite higher output coupling, mirror loss, and other potential electrodynamic losses.

The NTD $p$-Ge laser material was found to be a factor 1.3 to 1.6 times more highly compensated than the best of the melt-doped crystals studied for comparison. Nevertheless, the current density threshold for lasing was found to be 
considerably lower in the NTD crystal. This observation is consistent with the prediction from Monte Carlo calculations (Fig. 2) that compensation has little negative effect at hole concentration $3.6 \times 10^{13} \mathrm{~cm}^{-3}$ (the value for the NTD laser crystal tested.)

A controllable, repeatable process such as NTD should allow higher yield of good laser rods per $\mathrm{kg}$ of material. Given current prices for melt-doped $(\sim \$ 3 / \mathrm{g})$ and ultra-high purity Ge crystals $(\sim 5 / \mathrm{g})$, the yield need only be twice better to make NTD economically preferable. The neutronirradiation fee at UFTR is $\$ 100 / \mathrm{h}$, which is negligible compared with other costs of producing a commercial $p$-Ge laser (primarily cryogenics and specialized electronics). Residual radiation is equivalent to background, so properly aged NTD Ge poses no health risk. High-purity Ge starting material for NTD is commercially available while suitable melt-doped Ge is not a standard commercial item.

\section{SUMMARY AND CONCLUSIONS}

A far-infrared $p$-Ge laser with neutron transmutation doped active crystal was demonstrated. A high degree of uniformity for the Ga-acceptor distribution, easily obtained by NTD, but unusual in melt-doped Ge, was shown to be important for $p$-Ge laser operation. Current saturation behavior supports the expected higher homogeneity for this doping process. Despite the negative factor of higher compensation, this first unoptimized NTD $p$-Ge laser achieves a current density threshold significantly smaller than the best of 18 melt-doped lasers studied for comparison. Optimization of the NTD process should eventually permit smaller rods with lower thresholds, increased gain, and higher duty cycles.

\section{ACKNOWLEDGMENTS}

This work was partially supported by the National Science Foundation (ECS0070228), by a BMDO SBIR Phase I (administered by U.S. Army SMDC, DASG60-01-P-0043), and by an AFOSR STTR Phase II (F49620-02-C-0027). Neutron activation analysis was performed in Tashkent by $\mathrm{V}$. Z. Tsipin.

${ }^{1}$ E. Bründermann, in Long-Wavelength Infrared Semiconductor Lasers, edited by H. Choi (Wiley, New York, 2004), p. 279.

${ }^{2}$ R. C. Strijbos, J. G. S. Lok, and W. T. Wenckebach, J. Phys.: Condens. Matter 6, 7461 (1994).

${ }^{3}$ J. N. Hovenier, A. V. Muravjov, S. G. Pavlov, V. N. Shastin, R. C. Strijbos, and W. Th. Wenckebach, Appl. Phys. Lett. 71, 443 (1997).

${ }^{4}$ A. V. Muravjov, R. C. Strijbos, C. J. Fredricksen, H. Weidner, W. Trimble, A. Jamison, S. G. Pavlov, N. Shastin, and R. E. Peale, in Radiative Processes and Dephasing in Semiconductors, edited by D. S. Citrin (Optical Society of America, Washington, DC 1998) OSA-TOPS 18, 102 (1998).

${ }^{5}$ J. N. Hovenier, T. O. Klaassen, W. Th. Wenckebach, A. V. Muravjov, S. G. Pavlov, and V. N. Shastin, Appl. Phys. Lett. 72, 1140 (1998).

${ }^{6}$ A. V. Muravjov, R. C. Strijbos, C. J. Fredricksen, S. H. Withers, W. Trimble, S. G. Pavlov, V. N. Shastin, and R. E. Peale, Appl. Phys. Lett. 74, 167 (1999).
${ }^{7}$ A. V. Muravjov, S. H. Withers, R. C. Strijbos, S. G. Pavlov, V. N. Shastin, and R. E. Peale, Appl. Phys. Lett. 75, 2882 (1999).

${ }^{8}$ A. V. Muravjov, R. C. Strijbos, C. J. Fredricksen, H. Weidner, W. Trimble, S. H. Withers, S. G. Pavlov, V. N. Shastin, and R. E. Peale, Appl. Phys. Lett. 73, 3037 (1998)

${ }^{9}$ A. V. Murav'ev, I. M. Nefedov, S. G. Pavlov, and V. N. Shastin, Kvantovaya Elektron. (Moscow) 20, 142 (1993) [Sov. J. Quantum Electron. 23, 119 (1993)].

${ }^{10}$ A. V. Muravjov, S. H. Withers, H. Weidner, R. C. Strijbos, S. G. Pavlov, V. N. Shastin, and R. E. Peale, Appl. Phys. Lett. 76, 1996 (2000).

${ }^{11}$ E. W. Nelson, A. V. Muravjov, S. G. Pavlov, V. N. Shastin, and R. E. Peale, Infrared Phys. Technol. 42, 107 (2001).

${ }^{12}$ S. Komiyama, H. Morita, and I. Hosako, Jpn. J. Appl. Phys., Part 1 32, 4987 (1993)

${ }^{13}$ T. W. Du Bosq, R. E. Peale, E. W. Nelson, A. V. Muravjov, C. J. Fredricksen, N. Tache, and D. B. Tanner, J. Appl. Phys. 94, 5474 (2003).

${ }^{14}$ T. W. Du Bosq, R. E. Peale, E. W. Nelson, A. V. Muravjov, D. A. Walters, G. Subramanian, K. B. Sundaram, and C. J. Fredricksen, Opt. Laser Technol. (to be published).

${ }^{15}$ E. Bründermann, A. M. Linhart, H. P. Röser, O. D. Dubon, W. L. Hansen, and E. E. Haller, Appl. Phys. Lett. 68, 1359 (1996).

${ }^{16}$ R. C. Strijbos, Hole Transport Effects in p-Ge Lasers, Ph.D. dissertation, Technische Universiteit Delft, The Netherlands, 1997.

${ }^{17}$ A. V. Murav'ev, I. M. Nefedov, Yu. N. Nozdrin, and V. N. Shastin, Fiz. Tekh. Poluprovodn. (S.-Peterburg) 23, 1728 (1989) [Semiconductors 23, 1071 (1989)].

${ }^{18}$ Yu. A. Mityagin, V. N. Murzin, and S. A. Stoklitsky, Opt. Quantum Electron. 23, S287 (1991).

${ }^{19}$ E. Bründermann, A. M. Linhart, L. Reichertz, H. P. Röser, O. D. Dubon, W. L. Hansen, G. Sirmain, and E. E. Haller, Appl. Phys. Lett. 68, 3075 (1996).

${ }^{20}$ N. Hiromoto, I. Hosako, and M. Fujiwara, Appl. Phys. Lett. 74, 3432 (1999).

${ }^{21}$ S. V. Demihovsky, A. V. Murav'ev, S. G. Pavlov, and V. N. Shastin, Semicond. Sci. Technol. 7, B622 (1991).

${ }^{22}$ Yu. A. Mityagin, V. N. Murzin, O. N. Stepanov, and S. A. Stoklitsky, Semicond. Sci. Technol. 7, B641 (1991).

${ }^{23}$ A. V. Murav'ev, Yu. N. Nozdrin, S. A. Pavlov, and V. N. Shastin, Pis'ma Zh. Tekh. Fiz. 13, 65 (1987) [Sov. Tech. Phys. Lett. 13, 26 (1987)].

${ }^{24}$ F. Keilmann, V. N. Shastin, and R. Till, Appl. Phys. Lett. 58, 2205 (1991).

${ }^{25}$ F. Keilmann and H. Zuckermann, Opt. Commun. 109, 296 (1994).

${ }^{26}$ M. V. Dolguikh, A. V. Muravjov, and R. E. Peale, Proc. SPIE 5365, 184 (2004).

${ }^{27}$ C. J. Fredricksen, E. W. Nelson, A. V. Muravjov, and R. E. Peale, Infrared Phys. Technol. 44, 79 (2003).

${ }^{28}$ S. Shlimak, Phys. Solid State 41, 716 (1999).

${ }^{29}$ H. Fritzsche and M. Cuevas, Phys. Rev. 119, 1238 (1960).

${ }^{30}$ K. M. Itoh, E. E. Haller, W. L. Hansen, J. W. Beeman, J. W. Farmer, A. Rudnev, A. Tikhomirov, and V. I. Ozhogin, Appl. Phys. Lett. 64, 2121 (1994).

${ }^{31}$ E. W. Nelson, E. S. Flitsiyan, A. V. Muravjov, M. V. Dolguikh, R. E. Peale, S. H. Kleckley, W. G. Vernetson, and V. Z. Tsipin, Proc. SPIE 4993, 10 (2003).

${ }^{32}$ E. W. Nelson, M. V. Dolguikh, E. S. Flitsiyan, A. V. Muravjov, R. E. Peale, S. H. Kleckley, W. G. Vernetson, and V. Z. Tsipin, Proc. SPIE 5087, 133 (2003).

${ }^{33}$ E. Bründermann (private communication).

${ }^{34}$ L. E. Vorobjev, S. N. Danilov, and V. I. Stafeev, Opt. Quantum Electron. 23, S195 (1991)

${ }^{35}$ M. B. Prince, Phys. Rev. 92, 681 (1953).

${ }^{36}$ F. J. Morin and J. P. Maita, Phys. Rev. 94, 1525 (1954).

${ }^{37}$ E. W. Nelson, Ph.D. Dissertation, University of Central Florida, Orlando FL, 2003. 